\title{
A PERCEPÇÃO DE ACADÊMICOS DE ENFERMAGEM SOBRE A DISCIPLINA DE ANATOMIA HUMANA
}

\section{THE PERCEPTION OF NURSING ACADEMICS ABOUT HUMAN ANATOMY DISCIPLINE}

\author{
Rafaela Aparecida Rumpf Mildemberg" ${ }^{*}$, Gisele Gomes Reichel', Antônio Carlos Shwiderski' \\ 'Faculdades Integradas Santa Cruz de Curitiba/PR.
}

"Autor correspondente: Endereço: Rua Alfonso Osvaldo Grellmann, n¹35, Bairro: Pinheirinho; CEP: 81880-080; Curitiba-Pr. Telefone: (41) 99664-1442; E-mail: rafaela.rumpf@hotmail.com

\section{RESUMO}

Grande parte da prática profissional do enfermeiro está ligada, diretamente, ao cuidado com o corpo do paciente. Portanto, para saber cuidar, é preciso conhecimento básico sobre as suas estruturas e seu funcionamento. E essa base, para o desenvolvimento das ações de cuidado, é fornecida através de disciplinas como a Anatomia Humana. O objetivo deste trabalho foi avaliar a percepção dos acadêmicos de enfermagem quanto à importância da disciplina de Anatomia Humana, no curso de graduação em uma Faculdade privada de Curitiba. A metodologia utilizada nesta pesquisa foi um estudo descritivo transversal, com abordagem quantitativa de caráter exploratório, onde foi aplicado um questionário em vinte e cinco (25) discentes do curso de enfermagem, que já cursaram a disciplina de Anatomia Humana. Com o resultado, ficou evidenciado que todos os acadêmicos consideram a disciplina de Anatomia Humana importante, para a vida profissional do enfermeiro. Outro fato demonstrado foi o papel do professor como transmissor do conhecimento, a grande maioria dos participantes assinalaram que, tanto o conhecimento do assunto, como a metodologia e os recursos didáticos utilizados atuam, diretamente, sobre o aprendizado.

Palavras-chaves: História da anatomia; Anatomia e enfermagem; Ensino de anatomia.

\section{ABSTRACT}

Much of the nurse's professional practice is directly linked to caring for the patient's body. Therefore, to know how to take care, you need basic knowledge about its structures and functioning. And this basis, for the development of care actions, is provided through disciplines such as Human Anatomy. The objective of this work was to evaluate the perception of nursing students regarding the importance of the discipline of Human Anatomy, in the undergraduate course at a private Faculty in Curitiba. The methodology used in this research was a descriptive cross-sectional study, with an exploratory quantitative approach, where a questionnaire was applied to twenty-five (25) students of the nursing course, who have already studied the discipline of Human Anatomy. With the result, it was evident that all academics consider the discipline of Human Anatomy important, for the professional life of nurses. Another fact demonstrated was the role of the teacher as a transmitter of knowledge, the vast majority of participants pointed out that both the knowledge of the subject, as well as the methodology and didactic resources used act directly on learning. Key-words: Anatomy history; Anatomy and nursing; Teaching anatomy. 


\section{INTRODUÇÃO}

A Anatomia Humana é a ciência que estuda a morfologia do corpo humano, nomeando e descrevendo as suas estruturas nos níveis macroscópico e microscópico. Existem três principais métodos para estudo da anatomia: regional, sistêmica e clínica, sendo que a anatomia regional estuda o corpo por regiões, como tórax, abdome, cabeça entre outros. Já anatomia sistêmica estuda o corpo por sistemas, padronizado em sistema locomotor e sistema nervoso. A anatomia clínica é responsável pelo estudo da anatomia relacionada com as outras ciências da área de saúde (MOORE et. al., 2014).

Sendo uma disciplina comum para os cursos de enfermagem, medicina, odontologia, fisioterapia, entre outros, é cursada, geralmente, nos primeiros anos da formação, pois é importante para a compreensão de outras disciplinas como histologia, fisiologia, e outras, que no decorrer da graduação são exigidas no currículo básico desses cursos, por ser uma disciplina normativa e de evidente importância (CARDINOT et. al., 2014; CARVALHO, 2017).

O domínio do conhecimento das estruturas anatômicas não deve ser somente dos médicos, mas sim de todos os profissionais que cuidam dos pacientes, portanto, tal conhecimento é primordial para a formação do enfermeiro, pois este profissional busca planejar a prática dos procedimentos, de sua responsabilidade legal, em ambientes terapêuticos, através de princípios humanos, morfológicos e técnicos científicos (CARDINOT et. al., 2014).

Um componente expressivo para a formação acadêmica e profissional é o conhecimento do corpo que será tratado. Isso divulga, primordialmente, o eixo fundamental que conduz à prática da enfermagem, fornecendo recursos para uma discussão relacionada à compreensão morfológica, como ligação entre as disciplinas e a essência da área principal da enfermagem (CARVALHO, 2017).

O presente estudo torna-se relevante devido a disciplina de Anatomia Humana fazer parte do currículo da graduação em enfermagem, pois serve como base para o desenvolvimento profissional do enfermeiro e entendimento de outras disciplinas. O seu aprendizado é complexo devido à grande quantidade de estruturas e as terminologias utilizadas para descrevê-las, além do curto tempo para aprendê-las. Diante disso, se faz o seguinte questionamento: Qual a importância da disciplina de anatomia humana, para o curso de enfermagem, na percepção dos acadêmicos?

Para responder à problemática levantada neste estudo, o objetivo principal foi avaliar a percepção dos acadêmicos de enfermagem, quanto à importância da disciplina de Anatomia Humana no curso de graduação em uma Faculdade privada de Curitiba, bem como identificar os fatores que interferem no seu aprendizado e verificar as dificuldades dos acadêmicos frente ao seu aprendizado.

A disciplina de anatomia humana é uma disciplina básica para os cursos da área da saúde e, seu conhecimento, auxilia na compreensão de outras disciplinas fundamentais para o curso de enfermagem. Torna-se necessário que o enfermeiro tenha um bom conhecimento em anatomia para facilitar o desempenho de suas atividades técnicas, como por exemplo, uma simples punção de veia periférica que, quando não visível, necessita-se conhecer a anatomia dos vasos para localizá-los. Desta maneira, esse trabalho torna-se apropriado, já que possibilitará a constatação da opinião dos discentes de enfermagem, sobre a importância do conhecimento em anatomia para sua vida profissional. 


\section{METODOLOGIA}

O presente trabalho apresenta um estudo descritivo transversal, com abordagem quantitativa, de caráter descritivo e exploratório, aplicada em discentes de enfermagem que já tenham cursado a disciplina de Anatomia Humana. A pesquisa foi desenvolvida em uma Instituição de Ensino Superior (IES) particular na cidade de Curitiba/PR.

A pesquisa com abordagem quantitativa descritiva procura analisar os dados coletados, através da quantificação numérica, com aplicação de recursos e técnicas estatísticas para, fazer a descrição de uma população, fenômeno ou experiência, trazendo novas perspectivas da realidade já conhecida (DYNIEWICZ, 2014).

Para aprofundar os conhecimentos científicos sobre a importância da disciplina de Anatomia Humana para o curso de enfermagem, foi realizado um levantamento bibliográfico em livros, artigos, periódicos e materiais disponibilizados no Scientific Electronic Library Online (SCIELO), Google Acadêmico, na Biblioteca Virtual em Saúde (BVS) e na Biblioteca Virtual de Enfermagem (BVENF) dos últimos 5 anos (2014 a 2018).

Os participantes da pesquisa foram acadêmicos de uma IES, de ambos os sexos, e com faixa etária entre 18 e 50 anos, que se enquadrassem nos seguintes critérios de inclusão: estar matriculado na instituição, ser acadêmico do curso de enfermagem, e já ter cursado a disciplina de Anatomia Humana. Critérios de exclusão: ser acadêmico de outro curso da instituição referida, não ter concluído ainda a disciplina de Anatomia Humana, alunos afastados por licença maternidade ou doença.

Foram convidados a participar da pesquisa de forma voluntária todos os acadêmicos enquadrados no critério de inclusão. Os participantes que se voluntariaram e participaram foram um total de vinte e cinco (25) acadêmicos, os quais terão a garantia da preservação de seus dados e do seu anonimato. A pesquisa foi realizada pela própria pesquisadora, com a aplicação do questionário aos acadêmicos do Curso de Enfermagem que já tenham cursado a disciplina de Anatomia Humana, individualmente, em uma sala da IES, no turno da noite, em dias alternados.

O estudo foi realizado em uma IES através da aplicação de um questionário do tipo exploratório, objetivo e descritivo. A pesquisa realizou-se durante o mês de maio de 2018, após ser submetido à aprovação do Comitê de Ética em Pesquisa (CEP) em Seres Humanos do Instituto Paranaense de Otorrinolaringologia (IPO) - Curitiba/PR, sendo o Número do Parecer de Aprovação: 2.666.446.

Os dados coletados, a partir das respostas quantitativas do questionário, foram analisados e tabulados de forma estatística descritiva percentual e apresentados através de tabelas. Para o desenvolvimento do estudo, utilizaram-se os seguintes programas de computador como ferramenta o Microsoft Office Excel 2017 e o Microsoft Office Word 2017.

\section{RESULTADOS}

A amostra deste estudo foi composta por vinte e cinco (25) estudantes universitários, do curso de graduação de enfermagem, de uma IES, com idade entre 19 e 49 anos, que já tenham cursado a disciplina de Anatomia Humana.

No quesito sobre a importância da disciplina de Anatomia ao profissional enfermeiro, obteve-se como resultado a unanimidade dos participantes, evidenciando que todos os acadêmicos consideram a disciplina de Anatomia Humana importante para a vida profissional do enfermeiro. Outro fato 
demonstrado, foi que $56 \%$ destes acadêmicos declararam que não tiveram dificuldades durante o aprendizado da disciplina de Anatomia conforme apresentado na Tabela 1.

Tabela 1 - Distribuição dos resultados obtidos através de questionário respondido por acadêmicos de enfermagem de uma Instituição de Ensino Superior, Curitiba/PR, 2018.

\begin{tabular}{|c|c|c|c|c|}
\hline Questionamentos de Aprendizagem & Sim & Porcentagem (\%) & Não & Porcentagem (\%) \\
\hline $\begin{array}{l}\text { Importância da disciplina de Anatomia Humana } \\
\text { para a vida profissional enfermeiro }\end{array}$ & 25 & $100 \%$ & $* * *$ & $* * *$ \\
\hline Facilidade do aprendizado & 14 & $56 \%$ & 11 & $44 \%$ \\
\hline Existência de fatores que influenciam o aprendizado & 16 & $64 \%$ & 9 & $36 \%$ \\
\hline
\end{tabular}

Fonte: Autoria própria, 2018.

Embora nesse estudo realizado, tenha sido apontada a existência de fatores que interferem no aprendizado, 56\% dos participantes relataram que tiveram facilidade em aprender, sendo que os outros estudos revelam que é uma disciplina complexa devido à quantidade de termos.

Observa-se neste estudo, que dentre os fatores que influenciaram, positivamente, o aprendizado da disciplina de Anatomia Humana, todos os vinte e cinco (25) acadêmicos apontaram que ter curiosidade e interesse na disciplina, são fatores determinantes, para um bom desempenho no aprendizado. Outro fator importante, destacado pelos participantes, foi em relação à importância da disciplina de Anatomia Humana para o entendimento das demais disciplinas, no decorrer do curso de enfermagem, totalizando 80\% das respostas. Demais respostas podem ser vista na Tabela 2.

Tabela 2 - Distribuição dos resultados obtidos através de questionário respondido por acadêmicos de enfermagem de uma Instituição de Ensino Superior, Curitiba/PR, 2018.

\begin{tabular}{|c|c|c|c|c|}
\hline Fatores relacionados aos Acadêmicos & Sim & Porcentagem (\%) & Não & Porcentagem (\%) \\
\hline $\begin{array}{l}\text { Conhecimento prévio adquirido em cursos anteriores } \\
\text { (Téc. Enf.) }\end{array}$ & 13 & $52 \%$ & 12 & $48 \%$ \\
\hline Curiosidade e interesse no assunto. & 25 & $100 \%$ & $* * *$ & *** \\
\hline Contato contínuo com o ambiente escolar/acadêmico. & 15 & $60 \%$ & 10 & $40 \%$ \\
\hline $\begin{array}{l}\text { Disciplina e organização do tempo para os estudos e o } \\
\text { trabalho/vida pessoal. }\end{array}$ & 16 & $64 \%$ & 9 & $36 \%$ \\
\hline Importante para o entendimento de outras disciplinas. & 20 & $80 \%$ & 5 & $20 \%$ \\
\hline
\end{tabular}

Fonte: Autoria própria, 2018.

Quantos aos fatores que influenciaram no aprendizado dos acadêmicos entrevistados, da disciplina Anatomia Humana e o papel do professor como transmissor do conhecimento, a grande maioria dos participantes assinalou que tanto o conhecimento do assunto, como a metodologia e os recursos didáticos utilizados atuam, diretamente, sobre aprendizado, como se pode observar na Tabela 3.

TABELA 3 - Distribuição dos resultados obtidos através de questionário respondido por acadêmicos de enfermagem de uma Instituição de Ensino Superior, Curitiba/PR, 2018.

\begin{tabular}{l|l|l|l|l|l}
\hline Fatores relacionados ao Professor & Sim & Porcentagem (\%) & Não & \multicolumn{1}{l}{ Porcentagem (\%) } \\
\hline Conhecimento do assunto. & 23 & $92 \%$ & 2 & $8 \%$ \\
\hline Metodologia e recursos didáticos utilizados. & 24 & $96 \%$ & 1 & $4 \%$ \\
\hline
\end{tabular}

Fonte: Autoria própria, 2018. 
Ainda sobre os fatores que influenciam no aprendizado da disciplina de Anatomia Humana com qualidade, se observa que $72 \%$ dos participantes responderam que a carga horária deve ser adequada ao conteúdo ministrado, fator este muito importante para o curso de enfermagem. Segundo ponto mais assinalado pelos acadêmicos (72\%) foi o fato de considerarem a disciplina bastante complexa, isto devido ao seu grande número de termos técnicos usados, conforme descrito na Tabela 4.

Tabela 4 - Distribuição dos resultados obtidos através de questionário respondido por acadêmicos de enfermagem de uma Instituição de Ensino Superior, Curitiba/PR, 2018.

\begin{tabular}{|c|c|c|c|c|}
\hline Fatores determinantes para o aprendizado & Sim & Porcentagem (\%) & Não & Porcentagem (\%) \\
\hline Carga horária adequada ao conteúdo administrado. & 18 & $72 \%$ & 7 & $28 \%$ \\
\hline Intervalo adequado entre as avaliações. & 12 & $48 \%$ & 13 & $52 \%$ \\
\hline $\begin{array}{l}\text { Várias atividades associadas às avaliações que } \\
\text { compõem a nota. }\end{array}$ & 17 & $68 \%$ & 8 & $32 \%$ \\
\hline $\begin{array}{l}\text { Matéria complexa com grande número de termos } \\
\text { técnicos. }\end{array}$ & 18 & $72 \%$ & 7 & $28 \%$ \\
\hline Quantidade adequada de avaliações. & 14 & $56 \%$ & 11 & $44 \%$ \\
\hline
\end{tabular}

Fonte: Autoria própria, 2018.

Nos resultados pautados, referente aos fatores relacionados à estrutura de ensino, ficaram demonstrados que $76 \%$ dos acadêmicos consideram importante, para o aprendizado da disciplina de Anatomia Humana, ter um laboratório bem estruturado, com peças anatômicas em número suficiente e disponíveis para todos os alunos. Outra situação, levantada por $60 \%$ dos acadêmicos, foi o número de alunos em sala de aula e no laboratório, assim como, 56\% dos entrevistados considera que o acervo de livros na biblioteca deve ser em quantidade e variedade suficiente (Tabela 5).

Tabela 5 - Distribuição dos resultados obtidos através de questionário respondido por acadêmicos de enfermagem de uma Instituição de Ensino Superior, Curitiba/PR, 2018.

\begin{tabular}{|c|c|c|c|c|}
\hline Fatores relacionados à estrutura de ensino & Sim & Porcentagem (\%) & Não & Porcentagem (\%) \\
\hline Laboratórios e peças anatômicas em número suficiente. & 19 & $76 \%$ & 6 & $24 \%$ \\
\hline Excesso de disciplinas no mesmo período. & 9 & $36 \%$ & 16 & $64 \%$ \\
\hline Falta de monitoria para revisão das aulas práticas. & 12 & $48 \%$ & 13 & $52 \%$ \\
\hline $\begin{array}{l}\text { Biblioteca com acervo de livros em quantidade e } \\
\text { variado. }\end{array}$ & 14 & $56 \%$ & 11 & $44 \%$ \\
\hline Número de alunos em sala de aula e laboratório. & 15 & $60 \%$ & 10 & $40 \%$ \\
\hline
\end{tabular}

Fonte: Autoria própria, 2018.

Quando os acadêmicos foram indagados, para que apresentassem alguma sugestão ou alternativa para melhorar a qualidade e facilitar o aprendizado da disciplina de Anatomia Humana, evidenciaram-se os seguintes resultados: sugeriram aumentar a carga horária da disciplina; ter um laboratório melhor estruturado e mais espaçoso, com peças anatômicas reais e em número suficiente para todos; reduzir o número de alunos por turma; ter monitoria para auxiliar no aprendizado; melhorar o acervo de livros na biblioteca; e ter vídeo aulas complementando as aulas presenciais, de fácil acesso aos alunos (Tabela 6). 
Tabela 6 - Distribuição dos resultados obtidos através de questionário respondido por acadêmicos de enfermagem de uma Instituição de Ensino Superior, Curitiba/PR, 2018.

\begin{tabular}{|c|c|c|}
\hline Sugestōes para facilitar o aprendizado da disciplina & $\mathbf{N}^{\circ}$ & Porcentagem (\%) \\
\hline Aumentar carga horária da disciplina. & 7 & $28 \%$ \\
\hline $\begin{array}{l}\text { Laboratório: Ampliar espaço do laboratório; Aumentar } n^{\circ} \text { de peças anatômicas; } \\
\text { Utilizar peças anatômicas reais; Diminuir } n^{\circ} \text { de alunos por aula; Monitoria. }\end{array}$ & 13 & $52 \%$ \\
\hline Aumentar acervo de livros da biblioteca. & 2 & $8 \%$ \\
\hline Vídeo aulas. & 2 & $8 \%$ \\
\hline
\end{tabular}

Fonte: Autoria própria, 2018.

\section{DISCUSSÃO}

Os estudos, realizados por Salbego et.al. (2015) e Cardinot et. al. (2014), também apontam resultados parecidos com esses, pois na percepção de acadêmicos a disciplina de Anatomia é importante na formação do enfermeiro e serve de base para as outras ciências da grade curricular, visto que o seu aprendizado reflete, diretamente, no preparo para o desenvolvimento das atividades laborais desses profissionais.

Além disso, o estudo qualitativo de Salbego et.al. (2015) revela que algumas dificuldades encontradas durante o aprendizado dessa disciplina, se deve a grande variedade de termos, que denominam as diversas estruturas do corpo. Assim como, revela a existência de fatores que influenciam esse aprendizado, como por exemplo, a existência de laboratórios bem equipados.

No estudo de Ribeiro et. al. (2016), os autores apresentaram a complexidade dessa disciplina devido à sua variedade de conteúdo a ser ministrado e assimilado.

Corroborando com resultados acima citados, neste estudo, fica evidenciado o reconhecimento da importância dessa disciplina para atuação profissional do enfermeiro, visto que ela fornece o conhecimento das estruturas do corpo, possibilitando o planejamento do cuidado.

Mourthé Filho et. al. (2016) e Carvalho (2017) relatam em seus estudos que a disciplina de Anatomia Humana é importante para o entendimento de outras disciplinas. Enquanto que Borba (2017) e Cocce et. al. (2017) consideram a disciplina de Anatomia Humana fundamental para a introdução ao aprendizado de outras disciplinas, e este estudo corrobora com esta afirmação, pois mostra que 80\% dos entrevistados responderam que esta disciplina é importante para o entendimento de outras.

Assim como, neste estudo, ficou demonstrado que $64 \%$ dos acadêmicos relatam ter dificuldades em organizar o seu tempo para os estudos conciliando trabalho e vida pessoal, o estudo de Salbego et.al. (2015) apresenta resultado semelhante, pois revela que a maior parte dos estudantes trabalha e, portanto o tempo destinado aos estudos é reduzido.

Perante os resultados obtidos nesta pesquisa, observa-se que dentre os fatores que mais interferem no aprendizado dessa disciplina, os alunos destacaram a curiosidade e o interesse no assunto. Diante disso, percebe-se que esses fatores estão correlacionados, pois se o discente tiver interesse em aprender, organizará seu tempo para os estudos, corroborando com os estudos dos autores citados.

O resultado desta pesquisa mostrou que $92 \%$ dos acadêmicos consideraram que o conhecimento do professor é um fator importante para o aprendizado. Validando esse estudo, Mourthé Filho et. al. (2016) afirmam que a maneira com que o professor explica a matéria pode despertar maior interesse 
dos alunos no aprendizado, e Salbego et.al. (2015) dizem que, esse educador, além de ter o domínio da disciplina que ministra, necessita buscar metodologias inovadoras que estimulem o discente, não somente no aprendizado das terminologias anatômicas, mas também na capacidade de entendimento em relacionar essa ciência com a sua prática profissional.

O docente, além de utilizar metodologias que facilitem o aprendizado, tem um importante papel, que é o desafio de conseguir despertar o interesse dos alunos no assunto, fazendo com que, desta maneira, estes se dediquem mais aos estudos, revalidando estudos já realizados.

Conforme estudos, Salbego et.al. (2015) descrevem em sua pesquisa que a carga horária da disciplina é pequena diante da sua complexidade, o que dificulta o aprendizado, e, Nobeschi, Lombardi e Raimundo (2018) também apresentam, em seus resultados, a solicitação de uma carga horária maior para a disciplina de Anatomia Humana.

De acordo com Salbego et.al. (2015) os seus entrevistados sugerem que haja mais trabalhos para auxiliar o aprendizado do conteúdo e compor a nota dessa disciplina, assim como neste estudo que, $68 \%$ dos participantes assinalaram que acham importante a realização de várias atividades associadas às avaliações, para composição da nota.

Vindo de encontro com os resultados apresentados nesta pesquisa, a adequação da carga horária ao conteúdo ministrado, e a variedade de termos técnicos dessa matéria, são preocupações comuns entre os discentes, além disso, o estudo de Mourthé Filho et. al. (2016) corrobora revelando que a redução da carga horária dessa disciplina é uma aflição crescente entre o corpo docente.

Da mesma maneira que Cardinot et. al. (2014) expõem, em seu estudo, que o aprendizado desta disciplina é complexo, este trabalho reafirma essa questão, quando mostra o resultado obtido, no qual 72\% dos acadêmicos entrevistados assinalaram que essa matéria é complexa, devido ao grande número de termos técnicos que apresenta.

A pesquisa realizada por Salbego et.al. (2015) revela que as aulas práticas nos laboratórios são muito importantes para o aprendizado dessa disciplina. Semelhante foi o resultado desse estudo, o qual revelou que $76 \%$ dos estudantes consideram relevante, para os estudos, o uso de laboratórios com peças anatômicas em número suficiente, nas aulas práticas.

Assim como este estudo revela que $48 \%$ dos discentes acreditam que a falta de monitoria, para revisão das aulas práticas, afetam o seu aprendizado, o trabalho realizado por Cardinot et. al. (2014) apresenta resultado semelhante, descrevendo em sua pesquisa que $58 \%$ dos alunos consideram a monitoria importante para o aprendizado.

Diante dos resultados obtidos nesse estudo, se percebe que a utilização de laboratórios é importante para o ensino dessa disciplina, pois auxilia na assimilação do conteúdo, tornando-o mais dinâmico, já que é possível a visualização e a manipulação das peças anatômicas lá encontradas. Além disso, um bom acervo de livros, também é benéfico ao aprendizado, pois possibilita que o aluno visualize em atlas as estruturas do corpo em tamanhos aumentados e bem detalhadas.

Nessa pesquisa, surge a proposta de aumento da carga horária dessa disciplina para melhorar o aprendizado, o que corrobora com o estudo de Nobeschi, Lombardi e Raimundo (2018).

Uma das sugestões apresentadas neste estudo, pelos alunos, é a utilização de peças anatômicas reais, ou seja, de cadáveres. Porém Cocce et. al. (2017) relatam algumas desvantagens com essa prática, como a utilização de produtos tóxicos para a conservação, o desgaste devido à manipulação, entre 
outras, e sugere a utilização de novas tecnologias para suprir essa necessidade, como o uso de peças sintéticas, de softwares interativos, vídeos e multimídias, para auxiliar no aprendizado.

A pesquisa de Ribeiro et. al. (2016) e Foureaux et. al. (2018) aponta que a utilização de novas tecnologias favorece o ensino da disciplina de Anatomia. Portanto, um ambiente virtual de aprendizado complementa as aulas presenciais tradicionais, pois amplia os recursos didáticos, oferecendo mais meios aos estudos, podendo este ser realizado dentro e fora do ambiente acadêmico, corroborando com os achados desta pesquisa, onde os alunos apresentaram como sugestão a implementação de vídeos aulas.

Observa-se nessa pesquisa que pouco mais da metade dos alunos entrevistados sugerem melhorias relacionadas às aulas de laboratório, o que reafirma a relevância da utilização prática nas metodologias de ensino, para a facilitação no aprendizado da disciplina de Anatomia Humana.

\section{CONSIDERAÇÕES FINAIS}

Os objetivos dessa pesquisa foram alcançados, mostrando que a percepção dos acadêmicos foi unânime em relação à importância da disciplina de Anatomia Humana, na formação do profissional da saúde. Porém, foi identificado pelos acadêmicos os fatores que interferem no aprendizado e as dificuldades que eles encontram durante o processo de estudo.

Embora a disciplina de Anatomia Humana seja fundamental para a grade curricular, muitos acadêmicos apontaram dificuldade no aprendizado, e relacionaram como foco alguns fatores negativos, como a falta de conhecimento prévio da disciplina, a falta de tempo e organização dos estudos, e o não contato com o ambiente acadêmico. Outro fator interessante, respondido pela maioria dos acadêmicos, foi que a disciplina de Anatomia Humana é um pré requisito para o entendimento das demais disciplinas do currículo.

Além disso, com a realização desse estudo, pode-se constatar que, o papel do professor inspira o aprendizado dos acadêmicos, por ser o transmissor do conhecimento. Portanto, sua metodologia e os recursos didáticos são de grande valia para a qualidade da disciplina, influenciando, dessa forma, na capacitação do curso de enfermagem. A carga horária da disciplina deve ser apropriada com a quantidade do conteúdo ministrado, e as avaliações devam ser compostas por várias atividades na composição da nota, quesitos que mais se destacaram pelos acadêmicos entrevistados.

Os acadêmicos respaldam que a qualidade do ensino está diretamente ligada à disponibilidade e à utilização de laboratórios nas aulas práticas, com peças anatômicas suficientes para todos os alunos, e melhor distribuição de número de alunos nas aulas de práticas da disciplina.

Como apontado durante essa pesquisa, a disciplina de Anatomia Humana estuda a morfologia do corpo, fornecendo o conhecimento das suas estruturas e a base primária para outras disciplinas do curso. Dessa maneira, nesse estudo, foi abordada a percepção de acadêmicos do curso de enfermagem sobre essa disciplina, e embora haja tantos desafios que interferem no seu aprendizado, ficou evidenciado que todos os discentes de enfermagem, que participaram da pesquisa, reconhecem a sua importância para a sua futura atuação profissional.

Evidenciou-se, também, a importância do professor no aprendizado da disciplina de Anatomia, pois ficou notório que a metodologia adotada, o seu conhecimento no assunto e sua didática fazem 
despertar o interesse e o raciocínio nos alunos, proporcionando aulas mais interessantes e dinâmicas, correlacionando a disciplina e a futura profissão.

Ficou destacado pelos acadêmicos que sejam avaliadas as metodologias atuais, ajuste da grade horária, recursos físicos e materiais suficientes com monitoria para as aulas práticas, melhorar o acervo de livros disponíveis, a utilização de outros recursos inovadores, como vídeos com imagens 3D, tudo como uma forma de facilitação do aprendizado.

Espera-se que esse trabalho venha trazer uma reflexão, despertando o interesse sobre o tema, incentivando a realização de novos estudos. Fica a proposta de se fazer estudos comparativos com discentes que estão cursando a disciplina nos primeiros períodos e os discentes que estão finalizando o curso de graduação de enfermagem, para se ter uma visão mais ampla da importância da disciplina de Anatomia Humana. Também se faz necessário novas pesquisas que utilizem uma amostragem maior de alunos.

Fica a contribuição dos dados obtidos nessa pesquisa, para que as instituições e o corpo docente da disciplina de anatomia no curso de enfermagem possam fazer uso da melhor forma possível visando sempre a qualidade do aprendizado.

Admite-se a limitação dessa pesquisa, devido às dificuldades encontradas durante o levantamento bibliográfico, para desenvolver o estudo, pois embora o assunto tenha relevância para a vida profissional do enfermeiro, há carência de publicações atualizadas sobre o tema abordado.

\section{REFERÊNCIAS}

BORBA, K. P. DE. O estudo de anatomia no ensino de enfermagem: reflexões sobre Princípios éticos. Cienc. Cuid. Sau., v. 16, n. 1, jan.- mar., 2017.

CARDINOT, T. M. et. al. Importância da disciplina de anatomia humana para os discentes de enfermagem e farmácia da Abeu Centro Universitário de Belford Roxo/RJ. Coleção Pesquisa em Educação Física, Várzea Paulista, v. 13, n. 2, mar., 2014.

CARVALHO, C. A. F. Utilização de Metodologia Ativa de Ensino nas Aulas Práticas de Anatomia. Rev. Grad., USP, v. 2, n. 3, dez, 2017.

COCCE, A. L. R. et. al. O ensino da anatomia nas escolas de enfermagem: um estudo descritivo. Arq. Ciênc. Saúd., v. 24, n. 4, p. 08-13, out.-dez., 2017.

DYNIEWICZ, A. M. Metodologia da pesquisa em saúde para iniciantes. $3^{a}$ ed. São Caetano do Sul, São Paulo: Difusão, 2014. 247 p.

FOUREAUX, G. et. al. O ensino-aprendizagem da anatomia humana: avaliação do desempenho dos alunos após a utilização de mapas conceituais como uma estratégia pedagógica. Rev.

Ciênc. Educ., Bauru, v. 24, n. 1, p. 95-110, 2018.

MOORE, Keith L. et. al. Anatomia Orientada para a Clínica. $7^{a}$ ed. Rio de Janeiro: Guanabara Koogan, 2014. 1114 p.

MOURTHÉ FILHO, A. M. et. al. Refletindo o ensino da Anatomia Humana. Enf. Rev. v. 19, n. 2, p. 169$175,2016$. 
NOBESCHI, L.; LOMBARDI, L. A.; RAIMUNDO, R. D. Avaliação Sistemática da Dissecação como método de ensino e aprendizagem em Anatomia Humana. Rev. Eletron. Pesquiseduca . v. 10, n. 21, p. 420-432, 2018.

RIBEIRO, R. L. et. al. Desenvolvimento de objeto de aprendizagem para o ensino de Anatomia em Enfermagem. Rev. Rene., v. 17, n. 6, p. 866-873, Nov.-dez., 2016.

SALBEGO, C. et. al. Percepções Acadêmicas sobre o Ensino e a Aprendizagem em Anatomia Humana. Rev. Bras. de Ed. Méd., v. 39, n. 1, p. 23-31, 2015. 